VOL. $61(2000) \quad[459-462]$

\title{
ON $\omega$-LIMIT SETS OF AUTONOMOUS SYSTEMS IN INFINITE DIMENSIONAL BANACH SPACES
}

\author{
Gerd Herzog
}

\begin{abstract}
We prove that each Polish space is homeomorphic to the $\omega$-limit set of a bounded solution of an autonomous equation $x^{\prime}=f(x)$ in $l^{2} \times c_{0}$, in which $f$ is Lipschitz continuous on bounded sets.
\end{abstract}

\section{INTRODUCTION}

Let $(E,\|\cdot\|)$ be a real Banach space, let $f: E \rightarrow E$ be a continuous function and let $x:\left[t_{0}, \infty\right) \rightarrow E$ be a solution of $x^{\prime}=f(x)$. A point $\xi \in E$ is called $\omega$-limit point of $x$ if there is a sequence $\left(t_{k}\right)_{k=1}^{\infty}$ in $\left[t_{0}, \infty\right)$ with $t_{k} \rightarrow \infty$ as $k \rightarrow \infty$ such that $x\left(t_{k}\right) \rightarrow \xi$ as $k \rightarrow \infty$. The $\omega$-limit set of $x$ is defined by

$$
\omega(x):=\{\xi \in E: \xi \text { is } \omega \text {-limit point of } x\} .
$$

The set $\omega(x)$ is closed. If $\operatorname{dim} E<\infty$ and if $x$ is bounded then $\omega(x)$ is compact and connected (see for example [3, p.145]). If $x$ is unbounded, then $\omega(x)$ can be disconnected (see for example [8, p.343]). The topological structure of $\omega$-limit sets depends on the space dimension. For $\operatorname{dim} E=1$ any solution of $x^{\prime}=f(x)$ is monotone. Hence we have $\omega(x)=\emptyset$ or $\omega(x)=\left\{\lim _{t \rightarrow \infty} x(t)\right\}$. For $\operatorname{dim} E=2$ the Poincaré-Bendixson Theory (see for example [3, p.144]) gives some information on what $\omega(x)$ can look like. For $\operatorname{dim} E \geqslant 3$ less is known. Numerical plots of bounded solutions, for example of the Lorentz-equation (see for example [9, p.205]), indicate that $\omega$-limit sets can look quite weird. Moreover $\omega$-limit sets with topologically complicated structure are known (see for example [5] and the references given there). In infinite dimensions the $\omega$-limit set of a bounded solution can be empty or can be disconnected and/or not compact [6]. In [2] it is proved that given a closed separable set $P \subset E$ then there exists an autonomous system with continuous right hand side in $\mathbb{R} \times E$ and a solution $x$ of this system such that $\omega(x)=\{1\} \times P$ (in [2] limit sets are defined for $t \rightarrow 1$ - instead of $t \rightarrow \infty$ which is essentially equivalent; the right hand sides of the differential equations constructed in [2] are in general not Lipschitz continuous on bounded subsets of $\mathbb{R} \times E$ ).

Received February 28, 2000

The author wishes to express his sincere gratitude to Professor Dr. Roland Lemmert for helpful discussions improving the paper.

Copyright Clearance Centre, Inc. Serial-fee code: 0004-9727/00 \$A2.00+0.00. 
In this paper we shall prove that there is almost no topological restriction for $\omega$-limit sets in a certain infinite dimensional Banach space and for right hand sides which are Lipschitz continuous on bounded sets. If $E$ is separable, since $\omega(x)$ is closed we have that $\omega(x)$ is a Polish space. On the other hand we shall prove:

Theorem 1. Let $E=l^{2}(\mathbb{N}) \times c_{0}(\mathbb{N})$ and let $P$ be a Polish space. Then there exists a function $f: E \rightarrow E$ with the following properties:

1. $f$ is Lipschitz continuous on each bounded subset of $E$.

2. There is a bounded solution $x:\left[t_{0}, \infty\right) \rightarrow E$ of $x^{\prime}=f(x)$ such that $\omega(x)$ is homeomorphic to $P$.

\section{Proof of TheOREM 1}

In the sequel $\|\cdot\|_{2}$ denotes the Euclidean norm on $l^{2}(\mathbb{N})$ and $\|\cdot\|_{\infty}$ denotes the supremum norm on $c_{0}(\mathbb{N})$. Moreover let $(\cdot, \cdot)$ denote the standard inner product in $l^{2}(\mathbb{N})$.

SteP 1. (REDUCtion of THE PROBLEM). We shall only consider the case $P \neq \emptyset$. It is known [7] that each Polish space is homeomorphic to a closed subset of the unit sphere $\left\{\xi \in c_{0}(\mathbb{N}):\|\xi\|_{\infty}=1\right\}$. Since the unit spheres of two separable infinite dimensional Banach spaces are homeomorphic (see [1, p.188]) we can assume without loss of generality that $P$ is a closed subset of $\left\{\xi \in l^{2}(\mathbb{N}):\|\xi\|_{2}=1\right\}$.

The differential equation will be constructed in the space

$$
F=\mathbb{R}^{6} \times\left(l^{2}(\mathbb{N})\right)^{2} \times\left(c_{0}(\mathbb{N})\right)^{2}
$$

which is isomorphic to $E$.

SteP 2. (The UNIT SPHERE of $l^{2}(\mathbb{N})$ IS A LIMIT SET). Let $S: l^{2}(\mathbb{N}) \rightarrow l^{2}(\mathbb{N})$ denote the shift operator $S\left(\left(x_{n}\right)\right)=\left(x_{n+1}\right)$.

The operator $\exp (S)$ is hypercyclic (that is, it has dense orbits, see for example [4]) hence there exists $\xi_{0} \in l^{2}(\mathbb{N})$ such that $\left\{\exp (t S) \xi_{0}: t \geqslant 0\right\}$ is dense in $l^{2}(\mathbb{N})$. Now $h(t):=\left(\exp (t S) \xi_{0}\right) /\left\|\exp (t S) \xi_{0}\right\|_{2}, t \geqslant 0$ solves

$$
h^{\prime}=S h-(h, S h) h
$$

and $\omega(h)=\left\{\xi \in l^{2}(\mathbb{N}):\|\xi\|_{2}=1\right\}$. Moreover there exists $L>0$ such that $\| h(t)-$ $h(s) \|_{2} \leqslant L|t-s|, t, s \geqslant 0$.

Step 3. (The System AND THE solution). In the sequel let $A: c_{0}(\mathbb{N}) \rightarrow c_{0}(\mathbb{N})$ denote the linear operator $A\left(\left(x_{n}\right)\right)=\left(x_{n} / n\right)$ and let $\eta \in c_{0}(\mathbb{N})$ denote the sequence $\eta=(1 / n)$.

We consider the following differential equation (D) in $F$. 


$$
\begin{aligned}
u_{1}^{\prime} & =-u_{1}^{2} \\
u_{2}^{\prime} & =-u_{1} u_{2}^{2} \\
u_{3}^{\prime} & =-u_{1} u_{3}^{2} \\
u_{4}^{\prime} & =u_{1}(\operatorname{dist}(P, w)-\operatorname{dist}(P, v)) \\
u_{5}^{\prime} & =-u_{1} u_{6}(\operatorname{dist}(P, w)-\operatorname{dist}(P, v)) \\
u_{6}^{\prime} & =u_{1} u_{5}(\operatorname{dist}(P, w)-\operatorname{dist}(P, v)) \\
v^{\prime} & =u_{1} u_{2}(S v-(v, S v) v) \\
w^{\prime} & =u_{1} u_{3}(S w-(w, S w) w) \\
x^{\prime} & =(\operatorname{dist}(P, w)-\operatorname{dist}(P, v)) y+u_{6} \eta-A x \\
y^{\prime} & =-u_{1}^{2}(\operatorname{dist}(P, w)-\operatorname{dist}(P, v)) x+u_{1} u_{5} \eta-u_{1} y-A y .
\end{aligned}
$$

Obviously the right hand side of (D) is Lipschitz continuous on bounded subsets of $F$, and for $t_{0} \geqslant e$ the following function $z=(u, v, w, x, y):\left[t_{0}, \infty\right) \rightarrow F$ is a bounded solution of (D), as can be verified by differentiation.

$$
\begin{aligned}
& u_{1}(t)=1 / t, \quad u_{2}(t)=1 / \log (t), \quad u_{3}(t)=1 /(1+\log (t)) \\
& u_{4}(t)=\int_{\log (t)}^{1+\log (t)} \operatorname{dist}(P, h(\log (s))) d s, \quad u_{5}(t)=\cos \left(u_{4}(t)\right), \\
& u_{6}(t)=\sin \left(u_{4}(t)\right), \quad v(t)=h(\log (\log (t))), \quad w(t)=h(\log (1+\log (t))), \\
& x(t)=\left(x_{n}(t)\right)=\sin \left(u_{4}(t)\right)((1-\exp (-t / n)))_{n=1}^{\infty}, \\
& y(t)=\left(y_{n}(t)\right)=\frac{\cos \left(u_{4}(t)\right)}{t}((1-\exp (-t / n)))_{n=1}^{\infty} .
\end{aligned}
$$

STEP 4. (ThE $\omega$-LIMIT SET). Let $c=\left(c_{1}, c_{2}, c_{3}, c_{4}, c_{5}, c_{6}, c_{v}, c_{w}, c_{x}, c_{y}\right)$ be a limit point of $z$, and $\left(t_{k}\right)_{k=1}^{\infty}$ a corresponding sequence such that $z\left(t_{k}\right) \rightarrow c$ as $k \rightarrow \infty$. Note that $\left\|c_{w}\right\|_{2}=\left\|c_{v}\right\|_{2}=1$. Obviously $c_{1}=c_{2}=c_{3}=0$ and $c_{y}=0$. Moreover, by the mean value theorem

$$
\|w(t)-v(t)\|_{2} \leqslant L|\log (1+\log (t))-\log (\log (t))| \leqslant \frac{L}{\log (t)} .
$$

Hence $c_{w}=c_{v}$. Since dist $\left(P_{1} \cdot\right)$ is Lipschitz continuous with constant 1 we have

$$
\begin{aligned}
& \left|u_{4}\left(t_{k}\right)-\operatorname{dist}\left(P, c_{v}\right)\right| \\
& \quad=\left|\int_{\log \left(t_{k}\right)}^{1+\log \left(t_{k}\right)}\left(\operatorname{dist}(P, h(\log (s)))-\operatorname{dist}\left(P, c_{v}\right)\right) d s\right| \\
& \quad \leqslant \int_{\log \left(t_{k}\right)}^{1+\log \left(t_{k}\right)}\left\|h(\log (s))-h\left(\log \left(\log \left(t_{k}\right)\right)\right)+h\left(\log \left(\log \left(t_{k}\right)\right)\right)-c_{v}\right\|_{2} d s
\end{aligned}
$$




$$
\begin{aligned}
& \leqslant \int_{\log \left(t_{k}\right)}^{1+\log \left(t_{k}\right)} L\left(\log (s)-\log \left(\log \left(t_{k}\right)\right)\right) d s+\left\|v\left(t_{k}\right)-c_{v}\right\|_{2} \\
& \leqslant L\left(\log \left(1+\log \left(t_{k}\right)\right)-\log \left(\log \left(t_{k}\right)\right)\right)+\left\|v\left(t_{k}\right)-c_{v}\right\|_{2} \rightarrow 0
\end{aligned}
$$

as $k \rightarrow \infty$. Therefore $c_{4}=\operatorname{dist}\left(P, c_{v}\right)$. Next, dist $(P, \xi) \in[0,2]$ for $\|\xi\|_{2}=1$, hence $u_{4}(t) \in[0,2]$. Therefore $\left(x\left(t_{k}\right)\right)$ cannot converge in $c_{0}$ in the case that $c_{4} \neq 0$. For this reason we have $c_{4}=0$ (in particular $c_{v} \in P$ ) and from this we obtain $c_{5}=1, c_{6}=0$ and $c_{x}=0$. All together $c=\left(0,0,0,0,1,0, c_{v}, c_{v}, 0,0\right)$ which means that

$$
\omega(z) \subset Q:=\{(0,0,0,0,1,0, p, p, 0,0): p \in P\} .
$$

Now, let $p \in P$. According to Step 2. there is a sequence $\left(t_{k}\right)_{k=1}^{\infty}$ such that $h\left(\log \left(\log \left(t_{k}\right)\right)\right) \rightarrow p$ as $k \rightarrow \infty$. Then $v\left(t_{k}\right), w\left(t_{k}\right) \rightarrow p$ and $u_{4}\left(t_{k}\right) \rightarrow 0$ as $k \rightarrow \infty$. Hence $z\left(t_{k}\right) \rightarrow(0,0,0,0,1,0, p, p, 0,0)$ as $k \rightarrow \infty$.

Thus, we have $\omega(z)=Q$ and $Q$ is obviously homeomorphic to $P$.

\section{REFERENCES}

[1] C. Bessaga and A. Pełczyński, Selected topics in infinite-dimensional topology (Polish Scientific Publishers, Warszawa, 1975).

[2] B.M. Garay, ' $\omega$-limit sets in Banach spaces', in Colloq. Math. Soc. János Bolyai, Differential Equations and its Application 62 (North Holland, Amsterdam, 1991), pp. 139-158.

[3] P. Hartman, Ordinary differential equations (John Wiley and Sons, New York, London, Sydney, 1964).

[4] G. Herzog and C. Schmoeger, 'On operators $T$ such that $f(T)$ is hypercyclic', Studia Math. 108 (1994), 209-216.

[5] M.W. Hirsch, 'The dynamical system approach to differential equations', Bull. Amer. Math. Soc. 11 (1984), 1-64.

[6] E. Horst, 'Differential equations in Banach spaces: five examples', Arch. Math. (Basel) 46 (1986), 440-444.

[7] R. Kaufmann, 'Note on differential equations in Banach spaces', Complex Variables Theory Appl. 34 (1997), 141-144.

[8] V.V. Nemytskii and V.V. Stepanov, Qualitative theory of differential equations (Princeton University Press, Princeton, N.J., 1960).

[9] F. Verhulst, Nonlinear differential equations and dynamical systems (Springer-Verlag, Berlin, Heidelberg, New York, 1990).

Mathematisches Institut I

Universität Karlsruhe

D-76128 Karlsruhe

Germany

e-mail: gerd.herzog@math.uni-karlsruhe.de 